\title{
Echo of the quantum bounce
}

\author{
Luis J. Garay, ${ }^{1,2}$ Mercedes Martín-Benito, ${ }^{3}$ and Eduardo Martín-Martínez ${ }^{3,4,5}$ \\ ${ }^{1}$ Departamento de Física Teórica II, Universidad Complutense de Madrid, 28040 Madrid, Spain \\ ${ }^{2}$ Instituto de Estructura de la Materia, CSIC, 28006 Madrid, Spain \\ ${ }^{3}$ Perimeter Institute for Theoretical Physics, Waterloo, Ontario N2L 2Y5, Canada \\ ${ }^{4}$ Institute for Quantum Computing, University of Waterloo, Waterloo, Ontario N2L 3G1, Canada \\ ${ }^{5}$ Department of Applied Mathematics, University of Waterloo, Waterloo, Ontario N2L 3G1, Canada \\ (Received 20 August 2013; revised manuscript received 25 October 2013; published 13 February 2014) \\ We identify a signature of quantum gravitational effects that survives from the early Universe to the \\ current era: Fluctuations of quantum fields as seen by comoving observers are significantly influenced by \\ the history of the early Universe. In particular, we show how the existence (or not) of a quantum bounce \\ leaves a trace in the background quantum noise that is not damped and would be non-negligible even \\ nowadays. Furthermore, we estimate an upper bound for the typical energy and length scales where \\ quantum effects are relevant. We discuss how this signature might be observed and therefore used to build \\ falsifiability tests of quantum gravity theories.
}

DOI: 10.1103/PhysRevD.89.043510

PACS numbers: 98.80.Qc, 04.60.Pp, 04.62.+v

\section{INTRODUCTION}

In order to test whether a proposal for quantum gravity is correct or not, it is necessary to derive predictions and contrast them with observations. Cosmology might be one of the few (if not the only) accessible windows to observe quantum gravity effects, as they might be important for the early Universe, when matter densities approach Planck scales. In this paper we explore a way to assess the strength of the signatures of quantum gravity that might be observed nowadays.

Our proposal can be applied in general to find signatures of any early Universe deviations of standard general relativity (GR) i.e. post-Einsteinian corrections to the classical geometry. This said, as a first working scenario we concentrate on loop quantum cosmology (LQC) [1-3]. LQC adapts the methods of loop quantum gravity to quantize cosmological systems. Its main result is the replacement of the classical big bang singularity by a quantum bounce [4].

In the present study, we analyze the creation of particles measured by a particle detector due to the cosmological expansion when the surrounding matter fields are in the vacuum state. In cosmology this effect is known as the Gibbons-Hawking effect [5]. Since LQC and GR predict different dynamics for the early Universe, it is natural to ask whether the probability of excitation today for a hypothetical detector switched on since the early Universe is different for those two dynamics. In other words, we can ask whether there is any quantum signature in a system which has remained coupled to a field since the early Universe until the current era. These effects would have an imprint (via Gibbons-Hawking fluctuations and their power spectrum) in the cosmic microwave background (CMB). See e.g. [6]. Naively, one would expect that since the two dynamics (GR and LQC) are extremely similar for time scales beyond the Planck scale, there is little hope of finding such effects.

Remarkably, we show that these quantum-gravity signatures can survive until the current era with significant strength, being critically dependent on the specific model of early Universe physics. Let us consider as the cosmological background a flat, homogeneous, and isotropic space-time with compact spatial topology, and a homogeneous massless scalar as the matter source. This model is paradigmatic in LQC since it is solvable [7]. Interestingly, one can extract classical effective dynamics coming from this LQC quantum model [8], as it admits dynamical coherent states [9]. We will compare the effect of the LQC effective dynamics and that of GR on quantum fields.

With this aim, we introduce a test matter field which, for definiteness, we will choose to be a conformally coupled massless scalar $\phi$. We introduce an Unruh-DeWitt detector [10] coupled to $\phi$ that will be switched on at some instant of the early Universe when both LQC and GR dynamics are different. We shall show that the response of the detector at long times is different for LQC and GR and highly dependent on the early Universe physics, and the difference survives regardless of how long we wait, even though both dynamics are distinct only in the brief period when the energy density is of the order of the Planck scale. This will allow us to draw conclusions on the strength and model dependence of the signatures of quantum gravity that we could hope to detect in the current era.

\section{COSMOLOGICAL BACKGROUND DYNAMICS}

We consider a spatially flat, homogeneous and isotropic universe (FRW) with a massless scalar $\varphi$ as the matter source. The space-time geometry $\mathrm{d} s^{2}=-\mathrm{d} t^{2}+a^{2}(t) \mathrm{d} \boldsymbol{x}^{2}$ is 
characterized by a scale factor $a(t)$ (we will use natural units $c=\hbar=1$ ). As usual, for the canonical formalism to be well defined, we need to restrict the spatial manifold to a finite region [4]. For definiteness, we will consider a compact three-torus spatial topology, so that the spatial coordinates take values in a finite interval $[0, L]$, the physical volume of the universe being $[a(t) L]^{3}$. This spatial flat topology is compatible with the observational data about our Universe [6], as long as we consider the scale of compactification larger than the observable Universe.

The modified Friedmann equation [8]

$$
(\dot{a} / a)^{2}=\left(2 \ell_{\mathrm{P}}^{2} / 9\right) \rho\left(1-\rho / \rho_{\star}\right), \quad \rho_{\star}:=\ell_{\mathrm{P}}^{2} /\left(2 l^{6}\right)
$$

governs the classical effective dynamics obtained from the LQC model. Here, $\ell_{\mathrm{P}}=\sqrt{12 \pi G}$ is the Planck length; $\rho=\pi_{\varphi}^{2} /\left(2 a^{6} L^{6}\right)$, with $\pi_{\varphi}$ being the canonical momentum of $\varphi$, is the energy density of the field $\varphi ; l$ is a quantization parameter (in LQC the volume has a discrete spectrum equally spaced by $2 l^{3}$ units ${ }^{1}[1-3]$ ); and the critical density $\rho_{\star}$ is the maximum eigenvalue of the density operator in LQC [7]. In the limit $\rho_{\star} \rightarrow \infty$, or equivalently $l \rightarrow 0$, we recover GR. We will analyze how our results depend on this parameter $l . \pi_{\varphi}$ is a constant of motion whose value is typically chosen to be $\pi_{\varphi} \sim 10^{3}$ such that the dynamics admits an effective semiclassical description throughout the whole evolution [4].

The solution to the modified equation (1) reads

$$
a_{\mathrm{q}}(t)=\frac{l}{L}\left(\frac{\pi_{\varphi}^{2}}{\ell_{\mathrm{P}}^{2}}\right)^{1 / 6}\left[1+\left(\frac{\ell_{\mathrm{P}}^{2}}{l^{3}} t\right)^{2}\right]^{1 / 6} .
$$

The classical scale factor, $a_{\mathrm{c}}(t)=\lim _{l \rightarrow 0} a_{\mathrm{q}}(t)$, vanishes at $t=0$, and this leads to a big bang singularity. In contrast, the effective solution (2) never vanishes and takes a positive minimum value $a_{\mathrm{q}}(0) L=l\left(\pi_{\varphi}^{2} / \ell_{\mathrm{P}}^{2}\right)^{1 / 6}$ (see Fig. 1). In this case $-\infty<t<\infty$, the universe shrinks for $t<0$, bounces at $t=0$, and expands for $t>0$. The conformal time $\eta_{\mathrm{q}}$ in terms of the comoving time $t$ is given by an ordinary hypergeometric function:

$$
\eta_{\mathrm{q}}(t)=\frac{L}{l}\left(\frac{\ell_{\mathrm{P}}^{2}}{\pi_{\varphi}^{2}}\right)^{1 / 6}{ }_{2} F_{1}\left[\frac{1}{6}, \frac{1}{2}, \frac{3}{2},-\left(\frac{\ell_{\mathrm{P}}^{2}}{l^{3}} t\right)^{2}\right] t .
$$

In the limit $t \gg l^{3} / \ell_{\mathrm{P}}^{2}$, this function behaves as the classical one plus a constant, $\eta_{\mathrm{q}}(t) \rightarrow \eta_{\mathrm{c}}(t)+\beta$, where

\footnotetext{
${ }^{1}$ In the standard LQC literature $l$ is defined as $l:=(4 \pi G \gamma \sqrt{\Delta})^{1 / 3}$ [11], where $\gamma$ is the Immirzi parameter and $\Delta:=4 \pi \gamma \sqrt{3} l_{\mathrm{Pl}}^{2}$ is the minimum nonzero eigenvalue of the area operator of loop quantum gravity. In this way, $l$ is close to the Planck length $l_{\mathrm{Pl}}:=\sqrt{G \hbar}$.
}

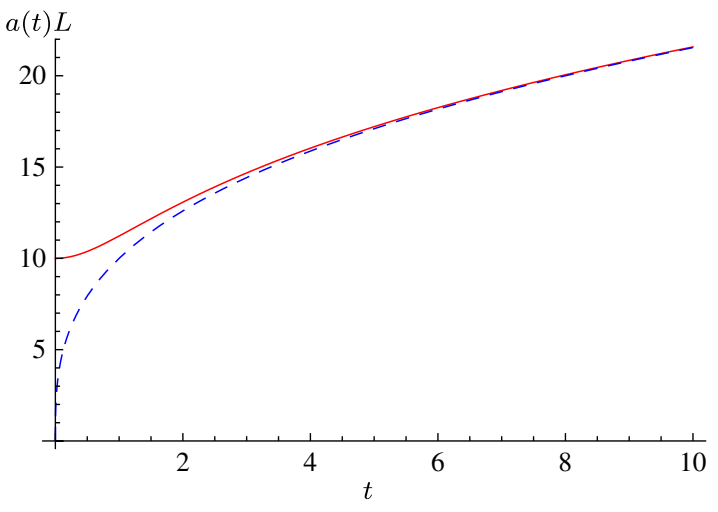

FIG. 1 (color online). Scale factor as a function of the proper time for $l=1, \pi_{\varphi}=1000$. The dashed blue curve represents the classical scale factor $a_{\mathrm{c}}(t) L$ and the solid red curve corresponds to the LQC effective scale factor $a_{\mathrm{q}}(t) L$. All quantities are expressed in Planck units, i.e. $\ell_{\mathrm{P}}=1$.

$$
\eta_{\mathrm{c}}(t)=\frac{3 L t^{2 / 3}}{2\left(\ell_{\mathrm{P}}^{2} \pi_{\varphi}^{2}\right)^{1 / 6}}, \quad \beta=\frac{l^{2} L \sqrt{\pi} \Gamma\left(-\frac{1}{3}\right)}{\left(\pi_{\varphi}^{2} \ell_{\mathrm{P}}^{10}\right)^{1 / 6} 2 \Gamma\left(\frac{1}{6}\right)} .
$$

Effective and classical dynamics are quite different at early times, while they agree at late times. A natural question arises: Can an observer distinguish-at late times-between both dynamics without having access to energy resources in the Planck scale? We will answer this question by analyzing the response of a low-energy detector coupled to a test field immersed in the above space-time.

\section{PARTICLE PRODUCTION IN COSMOLOGY}

We consider a conformal massless scalar field $\phi$ filling the volume of the universe. We Fock quantize this field choosing as vacuum the one associated with the following complete set of orthonormal modes for the conformally invariant 4-dimensional Klein-Gordon equation [12] in our cosmological background:

$$
u_{n}(\boldsymbol{x}, \eta)=\frac{e^{-\mathrm{i} \omega_{n} \eta}}{a(\eta) \sqrt{2 L^{3} \omega_{n}}} e^{\mathrm{i} \boldsymbol{k}_{n} \cdot \boldsymbol{x}} .
$$

Here, $\boldsymbol{k}_{\boldsymbol{n}}=\frac{2 \pi \boldsymbol{n}}{L}, \omega_{\boldsymbol{n}}=\left|\boldsymbol{k}_{\boldsymbol{n}}\right|$, and $\boldsymbol{n}=\left(n_{x}, n_{y}, n_{z}\right) \in \mathbb{Z}^{3}$. This vacuum remains invariant as the universe expands, and is usually referred to as "conformal vacuum", even though the field has a zero mode (see below). The field operator is $\hat{\phi}=\sum_{n}\left(\hat{a}_{n} u_{n}+\hat{a}_{n}^{\dagger} u_{n}^{*}\right)$ with $\hat{a}_{n}\left(\hat{a}_{n}^{\dagger}\right)$ the corresponding annihilation (creation) operators. This Fock quantization is the only one (up to unitary equivalence) with a vacuum invariant under the spatial isometries and unitary quantum dynamics [13,14]. In the above expression (5), we note that $\boldsymbol{n} \neq \mathbf{0}$. As discussed in $[13,14]$, in the following we will ignore the zero mode, and therefore in our calculations we 
assume that our detector is not coupled to this mode, restricting the sums to $\boldsymbol{n} \neq \mathbf{0}$. The unitary evolution and the uniqueness of the representation do not depend on the removal of a finite number of degrees of freedom. This mode can always be quantized separately. Furthermore, one can see that the coupling of the detector to the zero mode $\boldsymbol{n}=\mathbf{0}$ of the field is not relevant to the effects reported in this article. Something that can be readily seen by, for instance, introducing a small field mass or an IR regularization, or by coupling the detector to the derivative of the field instead of the field itself $[15,16]$ (a model which is free from IR divergencies).

In a homogeneous and isotropic universe there exists a family of privileged observers, called comoving observers, that see an isotropic expansion from their proper reference frame. Their proper time does not coincide with the conformal time. This means that comoving detectors actually detect particles even in the conformal vacuum defined above. This is the well-known Gibbons-Hawking effect [5].

A particle detector can be understood as a convenient witness of quantum fluctuations $[5,12]$. We will use the Unruh-DeWitt model [10], which describes the local monopole interaction of a two-level quantum system with a scalar field [12,17-19]. Although simple, this model encompasses all the fundamental features of the radiation-matter interaction [20]. The Hamiltonian of the coupled system in the interaction picture is $\hat{H}_{I}(t)=\lambda \chi(t)\left(\hat{\sigma}^{+} e^{i \Omega t}+\hat{\sigma}^{-} e^{-i \Omega t}\right) \hat{\phi}[\boldsymbol{x}(t), \eta(t)]$. Here, $\lambda$ is the coupling strength, $\chi(t)$ is the detector's switching function, $[\boldsymbol{x}(t), \eta(t)]$ is the world line of the detector, $\Omega$ is the energy gap between its ground and excited states, and $\hat{\sigma}^{ \pm}$are $S U(2)$ ladder operators.

We will consider several switching functions: namely sudden $\chi_{1}(t)$, linear ramping up $\chi_{2}(t)$, analytical ramping up $\chi_{3}(t)$, and analytically smooth activation $\chi_{4}(t)$, all of them compactly supported in the interval $t \in\left[T_{0}, T\right]$ :

$$
\begin{aligned}
& \chi_{1}(t)=1, \\
& \chi_{2}(t)= \begin{cases}\min \left[\left(t-T_{0}\right) / \delta, 1\right] & t<\left(T+T_{0}\right) / 2 \\
\min [(T-t) / \delta, 1] & t \geq\left(T+T_{0}\right) / 2,\end{cases} \\
& \chi_{3}(t)=\tanh \left(\frac{t-T_{0}}{\delta}\right)-\tanh \left(\frac{t-T}{\delta}\right)+\tanh \left(\frac{T_{0}-T}{\delta}\right), \\
& \chi_{4}(t)= \begin{cases}S\left[\left(t-T_{0}\right) / \delta\right] & t<T_{0}+\pi \delta \\
1 & t \in\left[T_{0}+\pi \delta, T-\pi \delta\right) \\
S[(T-t) / \delta] & t \geq T-\pi \delta,\end{cases}
\end{aligned}
$$

where $S(x)=[1-\tanh (\cot x)] / 2$ and $\delta$ controls the ramping up. Although for simplicity we consider a sudden switching $\chi_{1}(t)$ in several of our calculations, it is known that in $3+1 \mathrm{D}$ this leads to UV divergent integrals which only depend on the switching and not on the state of the field or the background geometry [21]. Nevertheless, as we will compute differences in probabilities of detectors with the same switching functions, our results will be devoid of any such switching effect (including UV divergences) and, furthermore, we show that our results are largely independent of the particular switching function.

We consider the test field to be in the conformal vacuum state defined above and the detector initially in its ground state. The detector is stationary in the comoving frame, $\boldsymbol{x}(t)=\boldsymbol{x}_{0}=\left(x_{0}, y_{0}, z_{0}\right)$. Provided that $\lambda$ is small enough, we can compute probabilities perturbatively. At leading order, the probability of transition for the detector, switched on at $T_{0}$, to be excited at time $T$ is

$$
\begin{gathered}
P_{\mathrm{e}}\left(T_{0}, T\right)=\lambda^{2} \sum_{n}\left|I_{\boldsymbol{n}}\left(T_{0}, T\right)\right|^{2}+\mathcal{O}\left(\lambda^{4}\right), \\
I_{\boldsymbol{n}}\left(T_{0}, T\right)=\int_{T_{0}}^{T} \mathrm{~d} t \frac{e^{-\frac{2 \pi i n \cdot x_{0}}{L}}}{a(t) \sqrt{2 \omega_{n} L^{3}}} e^{\mathrm{i}\left[\Omega t+\omega_{n} \eta(t)\right]},
\end{gathered}
$$

where the prime means that the $\boldsymbol{n}=\mathbf{0}$ mode is excluded from the sum.

\section{EXCITATION PROBABILITY OF THE DETECTOR UNDER BOTH SPACETIME DYNAMICS}

We will compare the probability $P_{\mathrm{e}}^{\mathrm{q}}\left(T_{0}, T\right)$ of the detector to get excited when the universe evolves under the effective LQC dynamics, with the probability $P_{\mathrm{e}}^{\mathrm{c}}\left(T_{0}, T\right)$ of the detector to get excited when the universe evolves under the GR dynamics. In particular we will check whether the signatures of the behavior at early times survive or not in the long time regime.

In order to alleviate the numerical computations, it is convenient to split the integrals in (8) into two intervals: $t \in\left[T_{0}, T_{\mathrm{m}}\right]$ and $t \in\left[T_{\mathrm{m}}, T\right] . T_{\mathrm{m}}$ is a short time sufficiently large for $\eta_{\mathrm{q}}\left(T_{\mathrm{m}}\right) \approx \eta_{\mathrm{c}}\left(T_{\mathrm{m}}\right)+\beta$ as shown in (4). In other words, we split the integrals in a regime where LQC and GR appreciably predict different dynamics and a regime of long times where the dynamics are essentially the same. $T_{\mathrm{m}}$ would typically be of the order of few times $l^{3} / \ell_{\mathrm{P}}^{2}$, as shown in Fig. 1. Then, the difference between the probabilities, $\Delta P_{\mathrm{e}}\left(T_{0}, T\right) \equiv P_{\mathrm{e}}^{\mathrm{q}}\left(T_{0}, T\right)-P_{\mathrm{e}}^{\mathrm{c}}\left(T_{0}, T\right)$, at leading order can be written as

$$
\begin{aligned}
\Delta P_{\mathrm{e}}\left(T_{0}, T\right)= & \lambda^{2} \sum_{n}{ }^{\prime}\left[\left|I_{\boldsymbol{n}}^{\mathrm{q}}\left(T_{0}, T_{\mathrm{m}}\right)\right|^{2}-\left|I_{\boldsymbol{n}}^{\mathrm{c}}\left(T_{0}, T_{\mathrm{m}}\right)\right|^{2}\right. \\
& +2 \operatorname{Re}\left(I _ { \boldsymbol { n } } ^ { \mathrm { c } * } ( T _ { \mathrm { m } } , T ) \left[e^{-\mathrm{i} \beta \omega_{n}} I_{\boldsymbol{n}}^{\mathrm{q}}\left(T_{0}, T_{\mathrm{m}}\right)\right.\right. \\
& \left.\left.\left.-I_{\boldsymbol{n}}^{\mathrm{c}}\left(T_{0}, T_{\mathrm{m}}\right)\right]\right)\right] .
\end{aligned}
$$

The difference of the detector's particle counting in both scenarios $\Delta P_{\mathrm{e}}\left(T_{0}, T\right)$ will be considerable even for $T \gg l^{3} / \ell_{\mathrm{P}}^{2}$, that is, if we look at the detector nowadays. Nevertheless, we would probably not be able to resolve 
times as small as $l^{3} / \ell_{\mathrm{P}}^{2}$ (roughly, the Planck scale). Instead, any observations we may make on particle detectors will be averaged in time over many Planck times,

$$
\left\langle P_{\mathrm{e}}\left(T_{0}, T\right)\right\rangle_{\mathcal{T}}=\frac{1}{\mathcal{T}} \int_{T-\mathcal{T}}^{T} P_{\mathrm{e}}\left(T_{0}, T^{\prime}\right) \mathrm{d} T^{\prime},
$$

where $\mathcal{T} \gg l^{3} / \ell_{\mathrm{P}}^{2}$ is the time resolution with which we can probe the detector. This will partially erase the observable difference between the response of the detector in the two regimes. Moreover, in order to remove any possible spurious effects coming from the big differences in the scales of the problem, we will consider a particle detector with an energy gap $\Omega \ll \ell_{\mathrm{P}}^{2} / l^{3}$, which additionally conspires against the hypothesized visibility of the effect. Remarkably, and contrary to intuition, the difference between the long time averaged response of sub-Planckian detectors in the GR and LQC scenarios remains nonnegligible even under these coarse-graining conditions.

Let us study how sensitive the response of the detector is to the LQC quantum parameter $l$ that characterizes the size of the quanta of volume. With this aim, let us consider a simple estimator: the mean of the relative difference between probabilities of excitation averaged over a long interval in the late time regime $\Delta T=T-T_{\text {late }}$, with $\Delta T$, $T_{\text {late }} \gg l^{3} / \ell_{\mathrm{P}}^{2}$ :

$$
E=\left\langle\frac{\left\langle\Delta P_{\mathrm{e}}\left(T_{0}, T\right)\right\rangle_{\mathcal{T}}}{\left\langle P_{\mathrm{e}}^{\mathrm{c}}\left(T_{0}, T\right)\right\rangle_{\mathcal{T}}}\right\rangle_{\Delta T}
$$

This estimator is well defined for all the switching functions above except for the sudden switching $\chi_{1}(t)$ [as it can be easily checked in formula (3.16) of [21]]. For this case, it is enough to replace the denominator by $P_{\mathrm{e}}^{\mathrm{c}}\left(T_{0}, T\right)_{\text {reg }}$, which is a UV regularized probability, defined by cutting up the sum in Eq. (7) to certain large frequency. This regularization is nonetheless qualitatively irrelevant: We could have used as the regularized denominator in Eq. (11) the difference with the probability of excitation of an identically switched detector in Minkowski spacetime, obtaining identical results. The UV cutoff regularization at the Planck scale provides a smaller value for the estimator so, for the sake of being as conservative as possible, we employ that one in Fig. 2(a). Note that, in a hypothetical experiment, one would measure the absolute difference between the experimentally observed response of the detector and a prediction for the very same detector based on a theoretical cosmological model. We make $E$ a relative difference estimator, however, to get an idea of the magnitude of the differences in the two models. It is easy to check that given that the vacuum excitation is always a bounded oscillatory function, the absolute difference behaves in a very similar way as the estimator defined above.
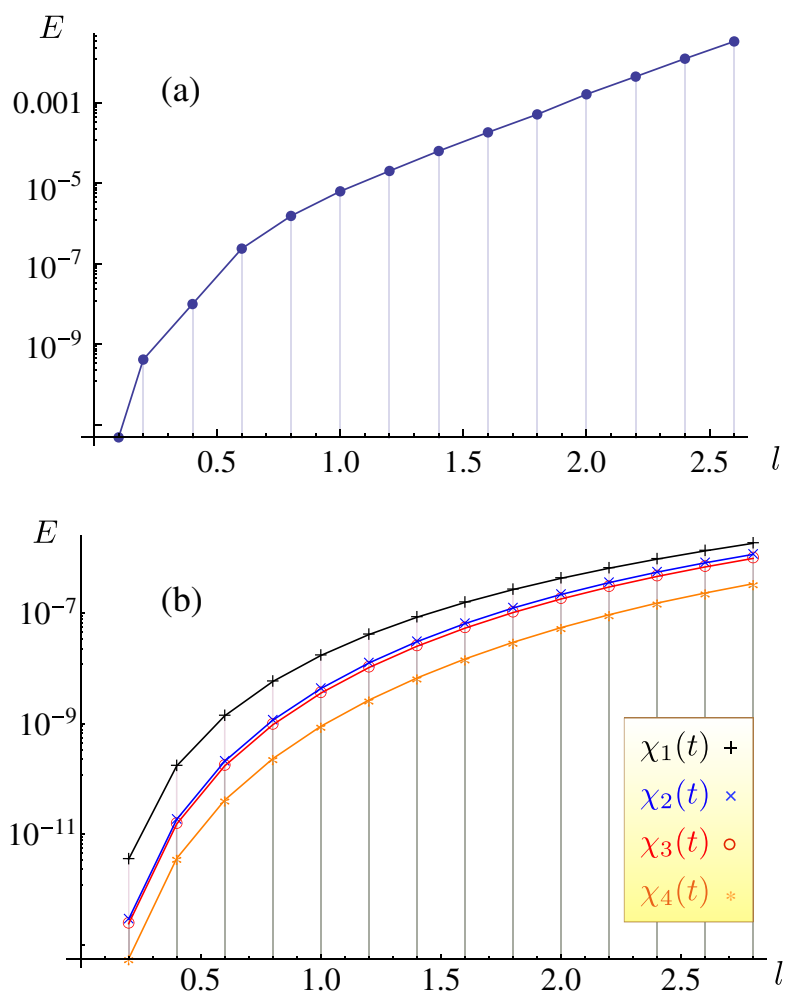

FIG. 2 (color online). (a) Logarithmic plot of the relative difference of the averaged probabilities $E$ (with a regularized denominator) for the sudden switching $\chi_{1}(t)$ as a function of the parameter $l$, for $\Omega \ll \ell_{\mathrm{P}}^{2} / l^{3}$ and $\pi_{\varphi}=1000$. The detector is switched on at $T_{0}=0.01$ (some early time after the bounce). (b) Logarithmic plot of the difference estimator $E$ as a function of the parameter $l$ for the different switching functions, for a switching time scale $\delta=10$ in $1+1 \mathrm{D}$. The behavior is identical in all four cases. All full-dimensional quantities are expressed in Planck units.

We will study $E$ as a function of the parameter $l$. This estimator tells us the difference in magnitude between the number of clicks of a detector in a LQC background and a detector in the classical background. As shown in Fig. 2(a), the variation of the response of the detector (the intensity of Gibbons-Hawking-type quantum fluctuations) grows exponentially with the size of the quantum of volume. This in turn means that the size of the quantum cannot be much beyond the Planck scale or the effects would be too large nowadays. Although this is a toy model, it captures the essence of a key phenomenon: Quantum field fluctuations are extremely sensitive to the physics of the early Universe, and the effect survives all the way to the current era. What is more important, this exponential dependence on the size of the volume quanta suggests that cosmological observations could put stringent upper bounds to the quantum scale $l$ in LQC or, equivalently, to the time scale $T_{\mathrm{m}}$ when the quantum effects become negligible. The exponential growth of the fluctuations with $l$ narrows its possible values to a range very close to the Planck scale. 
A very computationally demanding numerical calculation reveals that the same behavior observed for the sudden switching regime is also present in the case of the other smoother switchings $\chi_{2}(t), \chi_{3}(t), \chi_{4}(t)$. For computational convenience and to see how the dimension of space-time affects our result, we show in Fig. 2(b) the estimator $E$ in a $1+1 \mathrm{D}$ scenario for the different switchings. Note that in $1+1 \mathrm{D}$ the signal is overall smaller than in the $3+1 \mathrm{D}$. This indicates that the higher dimension amplifies the effect. The main result-exponential dependence of $E(l)$-remains for all four switching functions. In conclusion, the difference between the detector's averaged response in the classical and the quantum scenario depends exponentially on the scale of the quantum of volume and this exponential trend does not depend on the time scale of the detector's activation $\delta$ (smoothness of the switching) or the nature of the switching function.

\section{CONCLUSIONS}

We found that the Gibbons-Hawking effect is extremely sensitive to the physics of the very early Universe. A large difference appears between an effective model derived from LQC and the classical analog within GR, even if after some small time $T_{\mathrm{m}}$ both dynamics are indistinguishable. We could, in principle, think that the difference between probabilities might decay as the time $T$ during which the detector is switched on increases, since the larger $T$ is the longer both dynamics coincide. As we showed here, this is not the case and, most remarkably, the difference survives in time and is extremely sensitive to the specifics of the quantum theory, even under very conservative assumptions on the way that the fluctuations are detected. For instance, this may well allow for the derivations of stringent upper bounds on the quantum of volume that LQC displays.

Although we use the detector just as a witness of quantum fluctuations and the Gibbons-Hawking effect, one can think that generic nonconformal fields (massive fields, for instance) that couple to the test scalar field will undergo similar effects, therefore impacting inflation and the CMB. Actually, the analysis of inflation within LQC, and how quantum effects might affect the power spectrum of primordial fluctuations, has been recently considered [22-25]. Our study gives a different perspective on how to detect signatures of quantum gravity imprinted in the early Universe dynamics, and moreover serves to give an upper bound to the LQC quantum scale $l$.

This model can be generalized to the case of an infinitelevel harmonic oscillator detector whose response at leading order in perturbation theory is the same as that of the two level system [26,27]. Additionally, the choice of an initial vacuum other than the conformal vacuum used for our particular calculations would quantitatively change the results but one can see that this will not change the main result reported in this paper. Another extension of this work is the analysis of how signatures of quantum gravity can be enhanced by studying the quantum correlations acquired between two different detectors (e.g. exploring the entanglement-harvesting phenomenon; see e.g. [28]). Additionally, this work creates the basic tools to explore the possibility of transmission of quantum information from the prebounce shrinking universe to the expanding era in a LQC scenario, which will be studied elsewhere.

\section{ACKNOWLEDGMENTS}

We are indebted to J. Louko for his extremely helpful comments and sharp insight into the Unruh-DeWitt detector model. L. J. G. and M. M.-B. were partly funded by the MINECO Project No. FIS2011-30145-C03-02. L. J. G. was also funded by Consolider-Ingenio 2010: CPAN (CSD2007-00042). He also thanks the University of Waterloo and Perimeter Institute (PI) for their hospitality during his visit in 2012. E. M.-M. was supported by the Banting Fellowship Programme. Research at PI is supported by the Government of Canada through Industry Canada and by the Province of Ontario through the Ministry of Research and Innovation.
[1] M. Bojowald, Living Rev. Relativity 11, 4 (2008).

[2] K. Banerjee, G. Calcagni, and M. Martin-Benito, SIGMA 8, 016 (2012).

[3] A. Ashtekar and P. Singh, Classical Quantum Gravity 28, 213001 (2011).

[4] A. Ashtekar, T. Pawlowski, and P. Singh, Phys. Rev. D 74, 084003 (2006).

[5] G. W. Gibbons and S. W. Hawking, Phys. Rev. D 15, 2738 (1977).

[6] V. Mukhanov, Physical Foundations of Cosmology (Cambridge University Press, Cambridge, England, 2005).
[7] A. Ashtekar, A. Corichi, and P. Singh, Phys. Rev. D 77, 024046 (2008).

[8] V. Taveras, Phys. Rev. D 78, 064072 (2008).

[9] E. R. Livine and M. Martin-Benito, Phys. Rev. D 85, 124052 (2012).

[10] B. DeWitt, General Relativity; An Einstein Centenary Survey (Cambridge University Press, Cambridge, England, 1980).

[11] A. Ashtekar and E. Wilson-Ewing, Phys. Rev. D 79, 083535 (2009).

[12] E. Martín-Martínez and N. C. Menicucci, Classical Quantum Gravity 29, 224003 (2012). 
[13] L. C. Gomar, J. Cortez, D. Martin-de Blas, G. A. Mena Marugan, and J. M. Velhinho, J. Cosmol. Astropart. Phys. 11 (2012) 001.

[14] L. C. Gomar, J. Cortez, D. Martin-de Blas, G. A. M. Marugan, and J. Velhinho, arXiv:1212.5677.

[15] P. C. W. Davies and A. C. Ottewill, Phys. Rev. D 65, 104014 (2002).

[16] B. Juárez-Aubry and J. Louko, Detector for a massles field: Hawking effect without infrared sickness (unpublished).

[17] N. D. Birrell and P.C. W. Davies, Quantum Fields in Curved Space (Cambridge University Press, Cambridge, England, 1984).

[18] B. Reznik, A. Retzker, and J. Silman, Phys. Rev. A 71, 042104 (2005).

[19] E. Martín-Martínez, D. Aasen, and A. Kempf, Phys. Rev. Lett. 110, 160501 (2013).
[20] E. Martín-Martínez, M. Montero, and M. del Rey, Phys. Rev. D 87, 064038 (2013).

[21] J. Louko and A. Satz, Classical Quantum Gravity 25, 055012 (2008).

[22] M. Fernandez-Mendez, G. A. Mena Marugan, and J. Olmedo, Phys. Rev. D 86, 024003 (2012).

[23] M. Fernández-Méndez, G. A. Mena Marugán, and J. Olmedo, Phys. Rev. D 88, 044013 (2013).

[24] I. Agullo, A. Ashtekar, and W. Nelson, Phys. Rev. Lett. 109, 251301 (2012)

[25] A. Ashtekar, arXiv:1303.4989.

[26] E. G. Brown, E. Martín-Martínez, N. C. Menicucci, and R. B. Mann, Phys. Rev. D 87, 084062 (2013).

[27] D. E. Bruschi, A. R. Lee, and I. Fuentes, J. Phys. A 46, 165303 (2013).

[28] C. Sabín, B. Peropadre, M. del Rey, and E. Martín-Martínez, Phys. Rev. Lett. 109, 033602 (2012). 